\title{
NEW BOUNDS ON THE NUMBER OF BOUND STATES
}

\section{K. Chadan}

Laboratoire de Physique Théorique et Hautes Energies *) Université de Paris-Sud, 91405 Orsay

\author{
and \\ H. Grosse ${ }^{* *}$ ) \\ CERN -- Geneva
}

\section{ABSTRACT}

\begin{abstract}
We present methods for binding the number of bound states, which allow us to take into account oscillations of the potential. Previous results for a particular angular momentum and for levels below a certain energy are also improved.
\end{abstract}

*) Laboratoire associé au CNRS.

**) Permanent address : Institut für Theoretische
Physik, Universität Wien, A-1090 Wien, Austria. 


\section{INTRODUCTION}

There exist now many kinds of estimates on the number of bound states for a given potential with and without spherical symmetry 1)-7), as well as sufficient conditions for the existence of such states 8)-10). In these estimates usually either the attractive part of the potential or its absolute value enters.

In chaptex two of this paper we formulate the problem in such a way as to take into account oscillations of the potential, something which has not been done so far. For this purpose we introduce for the spherical symmetric case the following integral of the potential

$$
W_{\sigma}(r)=-\int_{r}^{\infty} d t \sigma^{2}(t) V(t)
$$

where $\sigma(t)$ will be chosen in a suitable way and. $W_{\sigma}(r)$ for $\sigma=1$ has already been used in scattering theory ${ }^{11}$ ), as well as for the bound state problem. Then, by making a special transformation, we arrive at an equivalent problem, as far as the number of bound states is concerned (i.e., the number of nodes of the zero energy wave functions), such that the new potential turns out to be everywhere attractive, whatever the sign of the starting potential may be. We then see that one can apply all estimates mentioned above, some of which are rather good, to this equivalent potential. On a simple example we shall see that our estimates give a large improvement over old results.

In the third part we transform the schrödinger equation into an integral equation with a symmetric kernel. Applying standard Birman-Schwinger techniques allows us to improve known results in two cases. On the one hand, an estimate on the number of bound states for a particular angular momentum also suitable for oscillating potentials is derived; on the other hand, states below a certain energy are estimated.

\section{BOUNDS FOR OSCIJJATING POTENTIALS}

Let us start with the schrödinger equation for a spherical symmetric potential; the reduced wave function for a bound state with energy $-\gamma^{2}$ and angular momentum $\ell$ fulfils

$$
\left[-\frac{d^{2}}{d r^{2}}+\frac{l(l+1)}{r^{2}}+8^{2}+V(r)\right] \varphi(r)=0, \varphi(0)=0
$$


$-2-$

where $V(r)$ should be such that $W_{\sigma}(x)$ defined in (1) fulfils, for $\sigma=1$ :

$$
W(r) \doteq W_{\sigma=1}(r) \in L^{1}([0, \infty)),\left.r W(r)\right|_{r=0, \infty}=0
$$

It has been shown ${ }^{11}$ ) that the whole of scattering theory (including the bound state problem), which works under the old Bargmann-Jost-Kohn-Levinson conditron

$$
r V(r) \in L^{1}([0, \infty))
$$

also works under the more general requirements (3). However, in this paper we shall keep (4) and leave the more general case for a future publication. Next we introduce

$$
U_{\sigma}(r)=\int_{r}^{\infty} d t \sigma^{-2}(t) W_{\sigma}(t)
$$

and make the following change of variables

$$
z(r)=\int_{0}^{r} d t \sigma^{-2}(t) e^{2 u_{\sigma}(t)}, \quad \psi_{\sigma}(z)=\left[\sigma^{-1}(r) e^{u_{\sigma}(r)} \varphi(r)\right] \int_{r=r(z)}(\sigma)
$$

where we take $\sigma(r)$ to be the solution of

$$
\left[-\frac{d^{2}}{d r^{2}}+\frac{l(l+1)}{r^{2}}+\gamma^{2}\right] \sigma(r)=0, \quad \gamma \geq 0, \sigma(r) \neq 0
$$

i.e.,

$$
\sigma(r)=i e^{i \pi l}\left[\frac{\pi}{2} i \delta r\right]^{1 / 2}(i \delta)^{l} H_{l+\frac{1}{2}}^{(1)}(i g r)
$$

where $\mathrm{H}_{v}^{(1)}$ is the Hanker function ${ }^{12)}$. Indeed, it is known that $\sigma(x)$ is real. ${ }^{12)}$, does not vanish for $r \geq 0, \quad y \geq 0, \ell>-\frac{1}{2}{ }^{13)}$, and we have

$$
\begin{aligned}
& \sigma(r)=(-)^{l}[(2 e-1) ! !] r^{-l}, \gamma \rightarrow 0 \\
& \sigma(r)=e^{-8 r}, e \rightarrow 0
\end{aligned}
$$


Note also that $|\sigma(r)|$ is a decreasing function of $r$ (it is $\infty$ at the oxigin, and 0 at $r=\infty$ ) as can be seen from the definition of Hankel function for $l=1,2, \ldots, 12)$.

$$
\text { Under (6), Eq. (2) transforms into }
$$

$$
\left[-\frac{d^{2}}{d z^{2}}+\widetilde{V}_{\sigma}(z)\right] \psi_{\sigma}(z)=0, \quad \tilde{V}_{\sigma}(z)=-\left.\left[W_{\sigma}^{2}(r) e^{-4 U_{\sigma}(r)}\right]\right|_{r=r(z)}(10)
$$

Notice, first of all, that the change of variables from $x$ to $z$ is
a $c^{2}$ bijection and maps the interval $[0, \infty)$ to $[0, \infty)$. Furthermore, it follows from the definition of $\psi_{\sigma}(z)$ [Eq. (6) that the nodes of $\phi_{\sigma}(z)$ and of $\varphi(r)$ correspond to each other. Therefore the number of bound states below energy $-\gamma^{2}$ of the original problem is equal to the number of nodes of $\psi_{\sigma}(z)$.

However, the surprising feature of Eq. (10) is that the new potential $\tilde{v}_{\sigma}(z)$ which enters is purely attractive. Moreover, using the monotonicity of $|\sigma(r)|$ as function of $r$ mentioned above, one can easily see, by using the inequality

$$
\int_{r}^{\infty} d t|W(t)| \leq \int_{r}^{\infty} d t|V(t)|(t-r)
$$

that

$$
\int_{0}^{\infty} d r r|V(r)|<\infty \quad \Rightarrow \quad \int_{0}^{\infty} d z z\left|\widetilde{V}_{\sigma}(z)\right|<\infty
$$

This means that $(10)$ is a good radial schrödinger equation in $z$ for the $s$ wave at zero energy with a purely attractive potential satisfying the Bargmann condition. This makes it clear that we can apply old results to (10) [and therefore to (2】 without being forced to throw out the repulsive part of the potential. So, for instance, we have the bounds on the number of bound states $n_{\ell}(\gamma)$ for angular momentum $l$ below energy $\left.-\gamma^{2} 3\right)$ :

$$
\begin{aligned}
& n_{e}(\gamma) \leq c_{p} \int_{0}^{\infty} \frac{d z}{z}\left(z^{2} \mid \tilde{V}_{\sigma}(z)\right)^{p}, \quad \forall p \geq 1 \\
& c_{p}=\frac{(p-1)^{p-1} \cdot \Gamma(z p)}{p^{p} \cdot \Gamma^{2}(p)}
\end{aligned}
$$

where $\sigma(r)$ entering in the transformation (6) is given by (8). 
$-4-$

To check the improvement over old results, we examine an example for $y=0, \quad l=0$ which means $\sigma(x)=1$.

Example :

Two $\delta$ - function potential. Let

$$
V(r)=\alpha \delta\left(r-r_{1}\right)+\beta \delta\left(r-r_{2}\right), r_{1}=1, r_{2}=2
$$

then it is trivial to obtain regions in $(\alpha, \beta)$ plane for which we have no bound states, respectively, one or two states. In order to apply (13), we have to calculate the new potential and work out the change of variables from $r$ to $z$; we obtain

$$
\tilde{V}_{\sigma}(z)=\left\{\begin{array}{cc}
-\frac{1}{4\left(z-K_{\alpha, \beta}\right)^{2}} & 0 \leq z \leq c_{\alpha, \beta}+\frac{e^{-2 \beta}}{2 \beta} \\
-\frac{1}{4\left(z-C_{\alpha, \beta}\right)^{2}} & c_{\alpha, \beta}+\frac{e^{-2 \beta}}{2 \beta} \leq z \leq c_{\alpha, \beta}+\frac{1}{2 \beta} \\
0 & c_{\alpha, \beta}+\frac{1}{2 \beta} \leq z
\end{array}\right.
$$

where the constants are given by

$$
K_{\alpha, \beta}=-\frac{e^{-2 \alpha-4 \beta}}{2(\alpha+\beta)} \quad, \quad C_{\alpha, \beta}=K_{\alpha, \beta}-\frac{e^{-2 \beta} \alpha}{2 \beta(\alpha+\beta)}
$$

The new variable clearly becomes asymptotically equal to $r$

$$
z(r)=\left\{\begin{array}{lc}
k_{\alpha, \beta} \cdot\left(1-e^{2(\alpha+\beta) r}\right) & 0 \leq r \leq 1 \\
c_{\alpha, \beta}+\frac{e^{-2 \beta(2-r)}}{2 \beta} & 1 \leq r \leq 2 \\
r-2+c_{\alpha, \beta}+\frac{1}{2 \beta} & 2 \leq r
\end{array}\right.
$$


In Table 1 (respectively, Table 2), we compare the naive estimate by taking

$$
|V(r)|_{-}=\theta(-V(r))|V(r)|
$$

and using Bargmann bound $[p=1$ in Eq. (13】] with results obtained with the help of $\widetilde{v}_{\sigma}(z)$ and taking $p=1$ or $\frac{3}{2}$. First, we fix $\alpha$ (respectively, $\beta$ ) such that the first bound state appears.

Then, using Eq. (13), we have determined bounds on $\beta$ (respectively, a) such that the number of bound states is zero. As expected, we obtain a large improvement over the naive estimates; the difference between our bounds and the exact results is of the order of a few pex cent.

\section{Remark}

Clearly the transformation (6) can be applied to the energy functional; one obtains

$$
E\left(\psi_{\sigma}\right)=\frac{\int_{0}^{\infty} d z\left[\left(\frac{d \psi_{\sigma}}{d z}\right)^{2}-\left.\left[\sigma^{4}(r) e^{-4 U_{\sigma}(r)} W_{\sigma}^{2}(r)\right]|| \psi_{r=r(z)} \psi_{\sigma}(z)\right|^{2}\right]}{\left.\int_{0}^{\infty} d z\left[\sigma^{4}(r) e^{-4 U_{\sigma}(r)}\right]\right|_{r=r(z)}\left|\psi_{\sigma}(z)\right|^{2}}
$$

Only in the case where $\left|\sigma(x) e^{-U_{\sigma}(x)}\right|_{r=r(z)} \leq 1$, there is a relation between the eigenvalues of the old problem to the eigenvalues of the operator

$$
-\frac{d^{2}}{d z^{2}}+\tilde{V}_{\sigma}(z) \text { on } L^{2}([0, \infty))
$$

\section{BOUNDS FOR ANGULAR MOMENTUM STATES BELOW SOME ENERGY}

In this part we shall show how to generalize to higher angular momentum and to negative energy some necessary conditions 4),5), as well as sufficient ones 10), found previously. To this end, we start from the equation 


$$
-\left(\frac{d}{d r}+\omega(r)\right) \sigma^{2}(r)\left(\frac{d}{d r}-\omega(r)\right) u(r)=\sigma^{2}(r) \mu(r) u(r), u(0)=0^{(21)}
$$

If we call now

$$
\sigma(r)\left(\frac{d}{d r}-\omega(r)\right) u(r)=\omega(r)
$$

we can easily invert $\mathrm{Eq}$. (22) and get

$$
u(r)=\int_{0}^{r} d t \sigma^{-1}(t) w(t) e^{\int_{t}^{r} d s \omega(s)}
$$

Using (23) we can transform the differential equation (21) into the integral equation

$$
w(r)=\int_{0}^{\infty} d s K^{(1)}(r, s) w(s)
$$

with a symmetric kernel

$$
K^{(1)}(r, s)=\int_{0}^{\infty} d t \theta(t-r) \theta(t-s) \frac{\sigma^{2}(t) \mu(t)}{\sigma(r) \sigma(s)} e^{\int_{s}^{t} d x \omega(x)+\int_{r}^{t} d x \omega(x)}
$$

It is also easily seen that if $\mu(t) \geq 0$ this kernel $K^{(1)}$ is positive definite.

Let us now specify $\sigma, \omega$ and $\omega$ to particular cases. To do so we take $\sigma$ from Eq. (8). The differential equation (21) turns under the substitution

$$
u(r)=\sigma^{-1}(r) \cdot \phi(r)
$$

into a Schrödinger equation for energy $-\gamma^{2}$ and angular momentum $\&$

$$
\left[-\frac{d^{2}}{d r^{2}}+\frac{\ell(\ell+1)}{r^{2}}+\gamma^{2}+\omega^{1}+\omega^{2}+\frac{2 \sigma^{\prime}}{\sigma} \omega-\mu\right] \phi(r)=0
$$

Iet $\mu(r)=-V(r)$ and take $\omega$ to be a solution of 


$$
\omega^{\prime}+\omega^{2}+\frac{2 \sigma^{\prime}}{\sigma} \omega=0, \quad \omega^{-1}(r)=\sigma^{2}(r) \int^{r} d t \sigma^{-2}(t)
$$

In case $\mathrm{V}$ is purely attractive, we now apply the Birman-Schwinger argument to $E q$. (24) and obtain a bound on the number of angular momentum $l$ bound states below energy $-\gamma^{2}, n_{\ell}(\gamma)$ of the form

$$
n_{e}(\gamma) \leq J_{r} K^{(1)}=\int_{0}^{\infty} d r V(r) \sigma^{2}(r) \int_{0}^{r} d t \sigma^{-2}(t) e^{2 \int_{t}^{r} d s \omega(r)}
$$

In the case where $V$ has no definite sign, we can iterate (24) once and use this iterated kemel $K^{(2)}$ which is now positive to get

$$
n_{e}(\gamma) \leq \operatorname{Jr} K^{(2)}
$$

A sufficient condition for the existence of bound states for purely attractive potentials is then given by 10 )

$$
\operatorname{Ir} K^{(2)} \geq \operatorname{Ir} K^{(1)}
$$

whereas for V's changing sign, we have to use

$$
\text { Ir } K^{(4)} \geq \operatorname{Ir} K^{(2)} \text {. }
$$

Let us quote two particularly interesting cases of the bounds given above :

A) For $\ell=0, \sigma(r)=e^{-\gamma r}$, we get $\omega(r)=2 \gamma$

$$
n_{0}(\gamma) \leq \pi K^{(1)}=\int_{0}^{\infty} d r e^{2 \gamma r} W_{\gamma}(r), W_{\gamma}(r)=-\int_{r}^{\infty} d r e^{-2 \gamma s} V(r)
$$

a result which is well known and due to schwinger ${ }^{2)}$. The next iterate gives

$$
n_{0}(\gamma) \leq \pi r K^{(2)}=\int_{0}^{\infty} d r e^{2 \gamma r} \frac{e^{2 \gamma r}-1}{8} W_{\gamma}^{2}(r)
$$


B) Choose $\gamma=0$ and $\sigma(r)=$ const. $r^{-l}$, then $\omega(r)=(2 \ell+1) / r$; we obtain first

$$
n_{l}(0) \leq \int_{0}^{\infty} d r W_{l}(r) /(2 l+1), W_{l}(r)=-\int_{r}^{\infty} d s s^{-2 l} V(s)
$$

The second iterate improves a result of Ref. 9):

$$
n_{l}(0) \leq \frac{2}{2 l+1} \int_{0}^{\infty} d r r^{4 l+1} W_{l}^{2}(r) \text {. }
$$

Remark :

It is interesting to note that the choice $\mu=\omega^{2}$ with $\omega$ being a solution of

$$
\omega^{\prime}+\frac{2 \sigma^{\prime}}{\sigma} \omega=V ; \sigma^{2}(r) \omega(r)=-\int_{r}^{\infty} d t V(t) \sigma^{2}(t)
$$

leads in Eq. (27) also to the Schrödinger equation for angular momentum $l$ and energy $-\gamma^{2}$. This time $\operatorname{TrK}{ }^{(1)}$ turns out to be identical to the Bargmann bound $[p=1$ of Eq. (13D), although the Birman-Schwinger technique is not applicable directly, because $\mathrm{K}^{(1)}$ does not depend linearly on $\mathrm{V}$.

\section{ACKNOWLEDGEMENTS}

One of us (H.G.) thanks the I.P.T.H.E. Orsay for a kind invitation; we both enjoyed the hospitality at CERN and thank V. Glaser for his interest and aiscussions. 
TABLES : The question of excluding bound states for the two $\delta$ - function potential

$$
V(r)=\alpha \delta\left(r-r_{1}\right)+\beta \delta\left(r-r_{2}\right), r_{1}=1, r_{2}=2
$$

is studied.

\begin{tabular}{|c|c|c|c|c|}
\hline$\alpha$ & $\beta_{\text {exact }}$ & $\beta_{\text {n.b. }}$ & $\beta_{\text {i.b. }}$ & $p$ \\
\hline-0.50 & -0.333 & -0.250 & -0.310 & 1 \\
-0.25 & -0.429 & -0.375 & -0.408 & 1 \\
0.25 & -0.556 & -0.500 & -0.544 & 1 \\
0.50 & -0.600 & -0.500 & -0.590 & 1 \\
0.75 & -0.636 & -0.500 & -0.624 & 1 \\
1.00 & -0.667 & -0.500 & -0.646 & 1 \\
\hline
\end{tabular}

TABLE 1 : We fix $\alpha$ and determine the exact value of $\beta$ such that there is a zero energy resonance ( $\beta$ exact $)$.
Next we determine a bound on $\beta$ excluding bound states, by taking the nažve estimate Eq. (18) and using Bargmann's result. $[\mathrm{p}=1$ in $\mathrm{Eq}$. (13L. These $\beta$. Tre compared to the improved $n_{b}$ ounds $\beta$ by using $\widetilde{\mathrm{V}}_{\sigma}(z)$ from Eq. (15) âna taking $p=1$ or $\frac{3}{2}$ in Eq. (13).

\begin{tabular}{|c|c|c|c|c|}
\hline$\beta$ & $\alpha_{\text {exact }}$ & $\alpha_{\text {n.b. }}$ & $\alpha_{\text {i.b. }}$ & $p$ \\
\hline-0.25 & -0.667 & -0.5 & -0.622 & 1 \\
0.25 & -1.200 & -1.0 & -1.158 & 1 \\
0.50 & -1.333 & -1.0 & -1.270 & 1 \\
0.75 & -1.429 & -1.0 & -1.368 & $\frac{3}{2}$ \\
1.00 & -1.500 & -1.0 & -1.411 & $\frac{3}{2}$ \\
\hline
\end{tabular}

TABIE 2: Same as in Table 1 but $\beta$ is fixed first and $\alpha$ values are determined. 


\section{REFERENCES}

1) V. Bargmann, Proc.Nat.Acad.Sci. (US) 38 (1952) 961.

2) M. Birman, Mat.Sb. 55 (1961) 125; AMS Trans. 53 (1966) 23;

J. Schwinger, Proc.Nat.Acad.Sci. (US) 47 (1961) 122.

3) V. Glaser, H. Grosse, A. Martin and W. Thirring, in "Studies in Mathematical Physics", Eds. E. Lieb, B. Simon and A. Wightman, Princeton University Press (1976), p.169.

4) K. Chadan, Lett.Math.Phys. 1 (1976) 281.

5) K. Chadan and A. Martin, Comm. Math.Phys. 53 (1977) 221.

6) G. RosenbIjum, Dokl.Akad. Nauk SSSR 202 (1972) 1012; Transl.Soviet Math. Dok1. 13 (1972) 245;

M. Cwickel, Ann. of Math. 106 (1977) 93;

E. Lieb, Bull.Amer.Math.Soc. 82 (1976) 751 and Proceedings 1979 AMS, Honolulu Conference.

7) G.C. Ghirardi and A. Rimini, J.Math.Phys. 6 (1965) 40.

8) F. Calogero, J.Math.Phys. 6 (1965) 161.

9) K. Chadan and A. Martin, J.Physique - Lettres 41 (1980) L205.

10) K. Chadan, in "Regards sur la Physique Contemporaine", Ed. R. Bacry CNRS, Paris (1980).

11) M.L. Baeteman and K. Chadan, Annales de I'I.H.P.A. XXIV (1976) 1.

12) A. Erdelyi, Higher Transcendental Functions, Vol.2, N.Y. (1953).

13) See Ref. 12), p.62. 